\title{
Aerodynamic and structural analysis of bio-mimetic corrugated wing
}

\begin{abstract}
This work includes the aerodynamic and static structural analysis of newly designed bio-mimetic corrugated aerofoil inspired from dragonfly forewing. Dragonfly corrugated aerofoil structure used in this research comprised of basal wing section located near the radius of the forewing. These corrugations define the stressed skin structure composed of grider-like veins structure and thin cuticle membrane which provides sophisticated mechanical advantage for resisting longitudinal bending while facilitating wing camber and torsion. Dragonflies are known for their impressive flight performance . They carry aerodynamic and inertial loads. A computational analysis is done on a newly designed dragonfly corrugated aerofoil at Reynolds number of 15632 , where flow is assumed to be laminar, steady, incompressible and two dimensional. The research comprises of the aerodynamic flow analysis of 2-D dragonfly corrugated aerofoil and static structural analysis using Ansys Fluent and Ansys Mechanical APDL. It is found that the design criteria being used and simulation carried out on corrugated aerofoil is giving much better results compared to earlier studies. The structural analysis also shows that it can resist maximum pressure loads and provides high rigidity to the span of the wing. These finding enhances the knowledge of insect inspired corrugated wing structure and facilitate the application for improved design of artificial wings for MAVs and UAVs.
\end{abstract}

Keywords: dragonfly, 2-d corrugated aerofoil, reynolds number, ansys fluent, ansys mechanical apdl, uavs, mavs
Volume 3 Issue 2 - 2019

\section{Md Akhtar Khan,' Chinmaya Padhy²}

'Department of Aerospace Engineering, GITAM University, India ${ }^{2}$ Department of Mechanical Engineering, GITAM University, India

Correspondence: Md Akhtar khan, Department of Aerospace Engineering, GITAM University, Hyderabad, India, Tel 63028I289I,Email khan.akhtar24@gmail.com

Received: May 25, 2019 | Published: June 26, 2019
Abbreviations: CFD, computational fluid dynamics; AoA, angle of attack

\section{Nomenclature}

$\rho$ Density of air, $\mathrm{kg} / \mathrm{m}^{3}$

v Free-stream velocity, $\mathrm{m} / \mathrm{s}$

$\mathrm{c}$ chord length of the aerofoil, $\mathrm{m}$

$\alpha$ Angle of attack

$\mu$ Dynamic viscosity, Pa.s

L Lift force, $\mathrm{N}$

D Drag Force, $\mathrm{N}$

$\mathrm{C}_{\mathrm{L}}$ Lift Coefficient

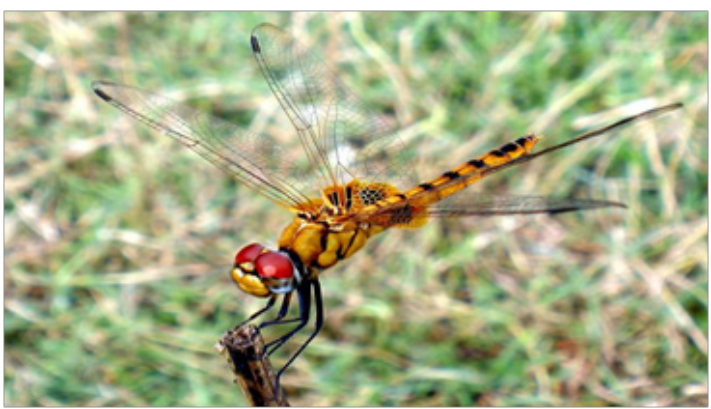

(a)
$\mathrm{C}_{\mathrm{D}}$ Drag Coefficient

Re Reynolds Number $[\rho \mathrm{Vl} / \mu]$

\section{Introduction}

The approach can unlock the design principles of the wings of one of nature's most remarkable aeronauts, the dragonfly. Dragonfly wings are complex biological composite structures and consist of two main structural components, an ultra thin membrane supported by reinforcing hollow veins. In the present study we used ANSYS-15 for computational aerodynamics analysis and structural analysis of newly designed corrugated structure inspired from the forewing of 'Pantala flavescens' dragonfly. Dragonfly being a natural flyer the vein and the membrane structure are flexible in nature and it is difficult to predict the behavior of dragonfly corrugated wing during flight condition as shown in Figure 1a and Figure 1b.

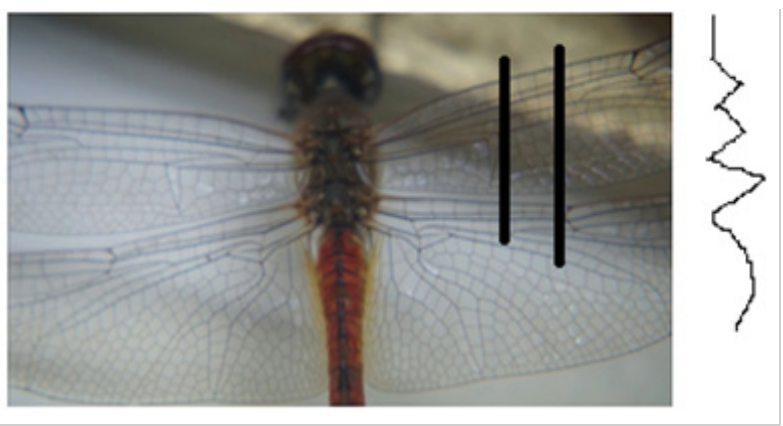

(b)

Figure I (a) Pantala flavescens dragonfly (b) Pantala flavescens dragonfly corrugated wing vein structure. 
The dragonfly corrugated airfoil used in this study is wavy in nature (i.e. consists of valley) and has the chord length of $110 \mathrm{~mm}$. The corrugated design is with increasing corrugation angles with increasing peak heights and having a curved profile at the trailing edge as shown in Figure 2. ${ }^{1}$

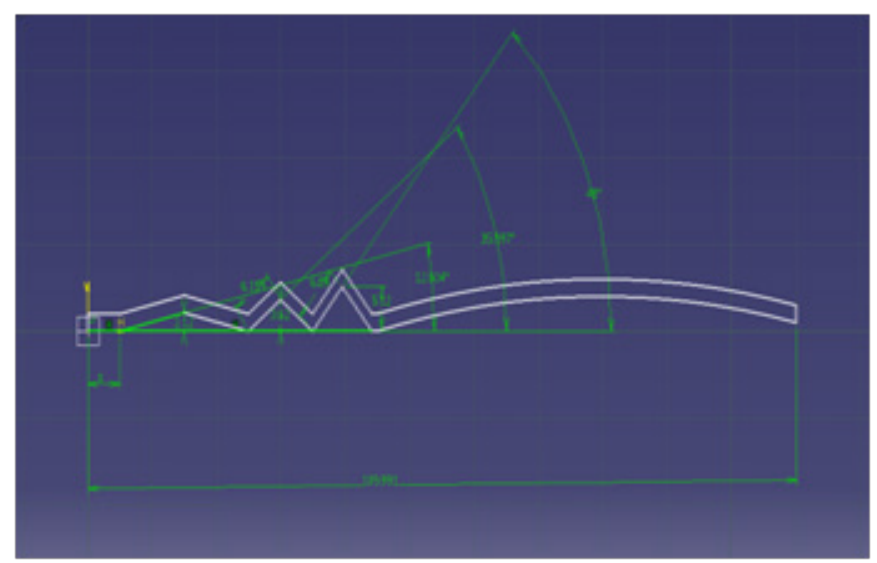

Figure 2 2-D corrugated aerofoil.

$\mathrm{Kesel}^{2}$ noticed trapped vortices present in the folds that serve to change the effective profile of the airfoil. With the advent of bioinspired aerial vehicle, it has become clear that there is much that can be learnt from insect flight that could be translated into engineered systems. The negative pressure developed in these corrugations contributes to the excessive lift.

Okama et al., ${ }^{3}$ isolated the influence of different geometrical parameters and showed that the surface texture or the roughness of wings results in an increased maximum lift coefficient, $\mathrm{C}_{\mathrm{Lmax}}$ and increases maximum lift to drag ratio $(\mathrm{L} / \mathrm{D})_{\max }$.

Dragonfly wings shows complete corrugation with the stems of the main longitudinal veins and the composite veins are linked at the nodus. In addition, reinforcement can be provided by the cross-veins of the nodus. Nodus prevents fracture on the vein structure during flight. ${ }^{4}$

\section{CFD domain and methodology}

The resolution of the grids around the wing is particularly important as it determines the accuracy of the analysis. Aerodynamic performance of corrugated aerofoil is carried out by using structure grid Navier-stokes method. The size of the grid that comes in contact with wing is determined as $1 \%$ of the boundary layer thickness at the wing trailing edge Table 1 Computational domain (Figure $3 \& 4$ ).

Table I Computational domain

\begin{tabular}{|c|c|}
\hline Domain size & $910 \times 600 \mathrm{~mm}$ \\
\hline Number of nodes & 434230 \\
\hline Min orthogonality & 0.511 \\
\hline Velocity & $2.1 \mathrm{~m} / \mathrm{s}$ \\
\hline Dynamic viscosity $(\mu)$ & $1.789 \times 10^{-5}$ \\
\hline Density & $1.225 \mathrm{~kg} / \mathrm{m}^{3}$ \\
\hline
\end{tabular}

$$
\begin{aligned}
& \frac{\partial}{\partial x_{j}}\left(u_{i} u_{j}\right)=-\frac{\partial p}{\partial x_{i}}+\frac{1}{\operatorname{Re}} \frac{\partial^{2} u_{i}}{\partial x_{i} \partial x_{j}} \\
& \operatorname{Re}=\frac{\rho v c}{\mu} \\
& C_{L}=\frac{L}{0.5 \rho_{v}^{2} S} \\
& C_{D}=\frac{D}{0.5 \rho_{v}^{2} S}
\end{aligned}
$$

where, $\mathrm{s}=\mathrm{b} \times \mathrm{c}$, for 2 -D wing $\mathrm{b}=1$

$$
\text { Gliding Ratio }=\frac{C_{L}}{C_{D}}=\frac{L}{D}
$$

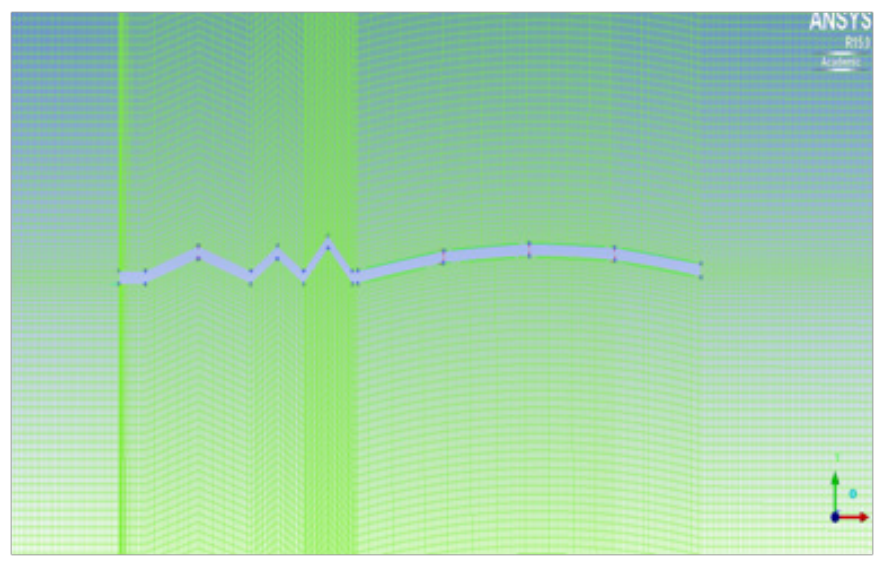

Figure 3 Computational grid.

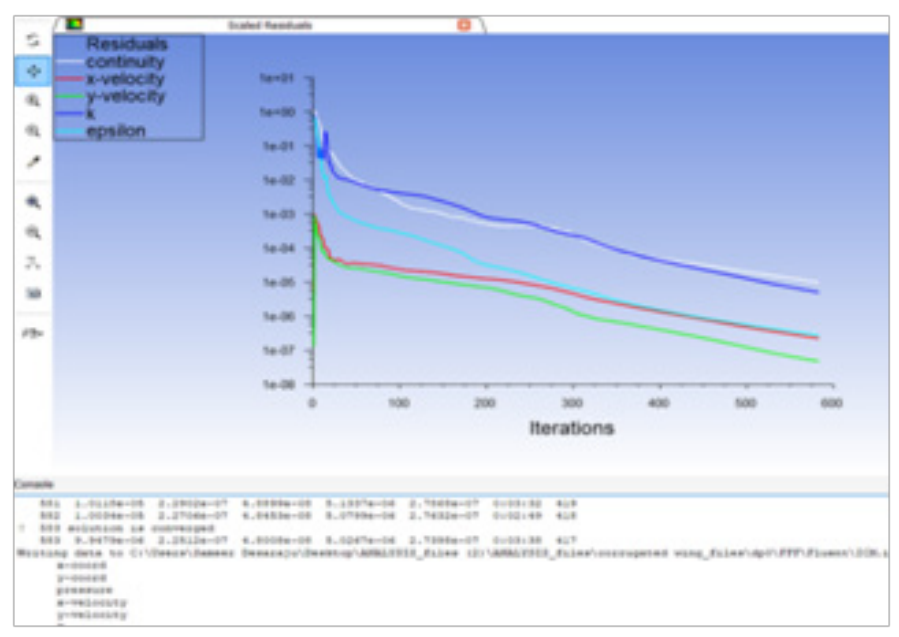

Figure 4 Convergence model for corrugated wing.

\section{CFD analysis results of corrugated wing}

We have seen that velocity is maximum at the peaks and minimum in the valleys and pressure is maximum at the stagnation point as illustrated in Figure 5a and Figure 5b. The beauty of this design is that 
even at zero degree angle of attack the vortices are trapped inside the valleys as shown in Figure 5c which helps in delaying flow separation. Instead as the angle of attack of the corrugated wing increases from $2^{\circ}$ to $8^{\circ}$ as shown in Figure 5 e to Figure $5 \mathrm{j}$ the corresponding lift increases which increases the aerodynamic performance of the wing indicates the suitability of the pleated corrugated aerofoil for MAVs and UAVs. The stalling angle for this corrugated wing is in the range of $6^{\circ}-8^{\circ}$ where lift starts decreasing gradually. ${ }^{5}$

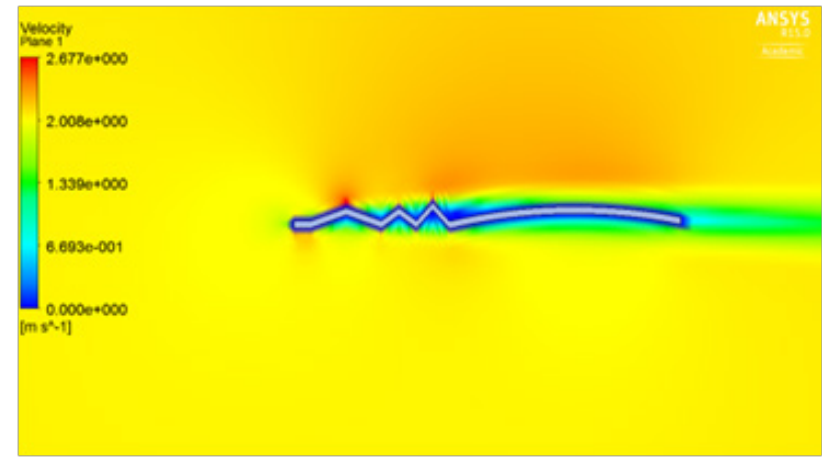

(a)

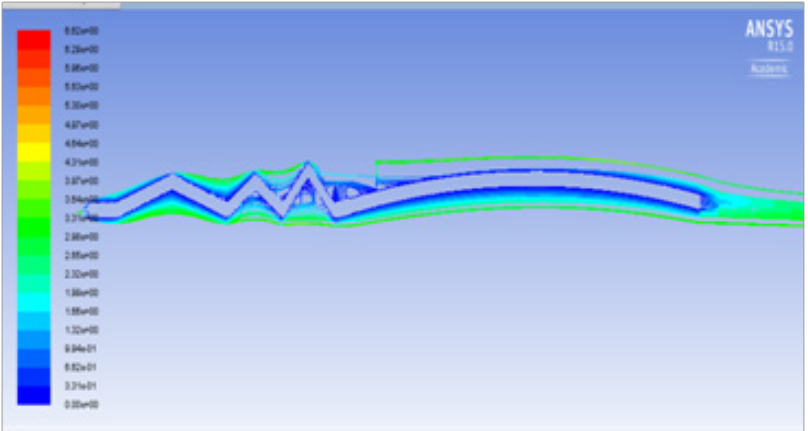

(c)

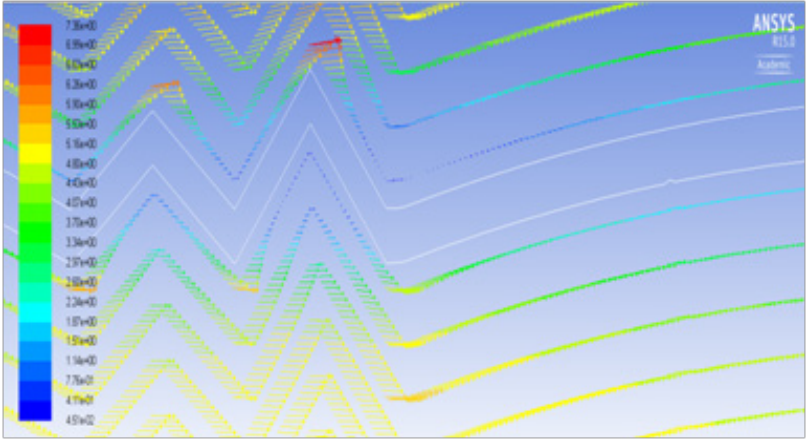

(e)

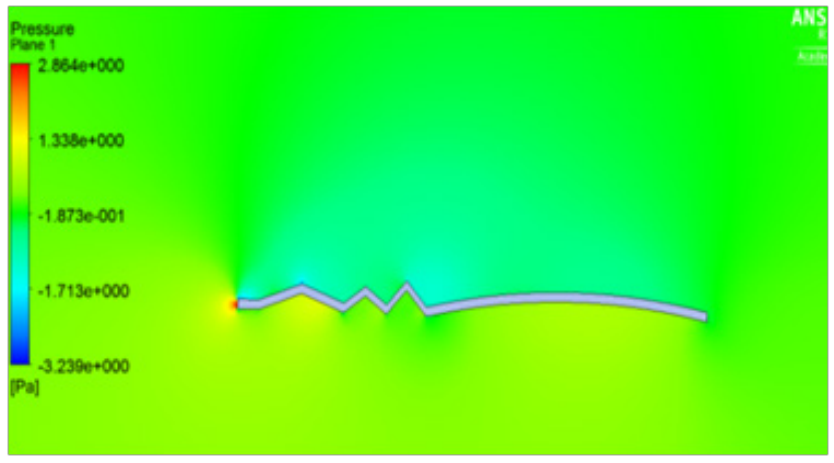

(g)

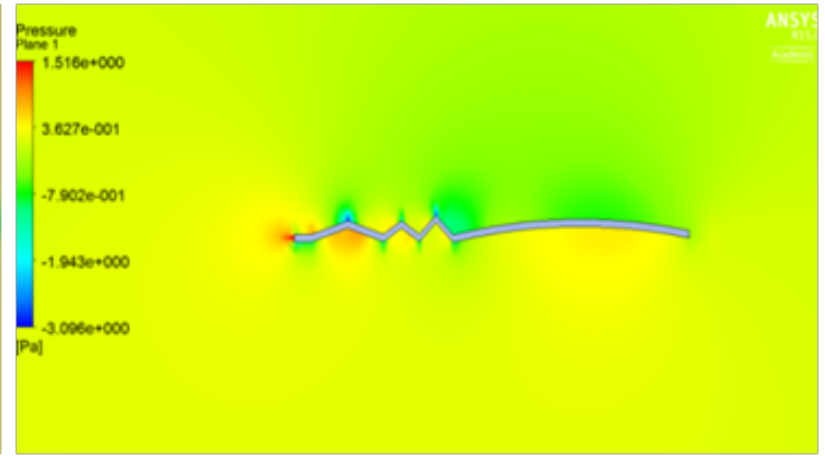

(b)

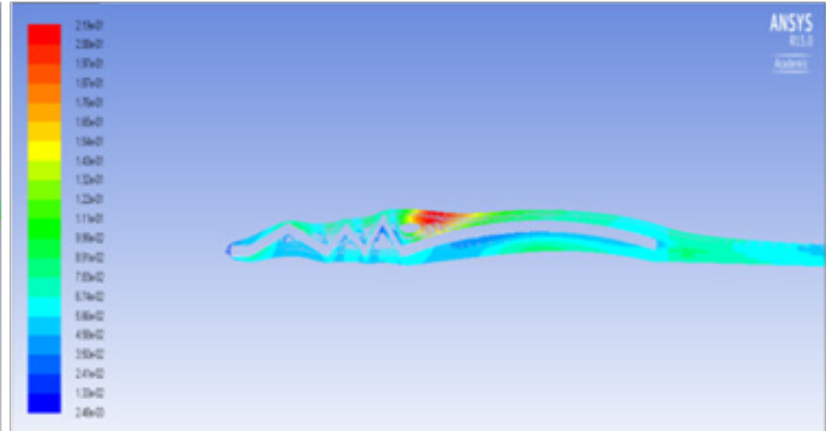

(d)

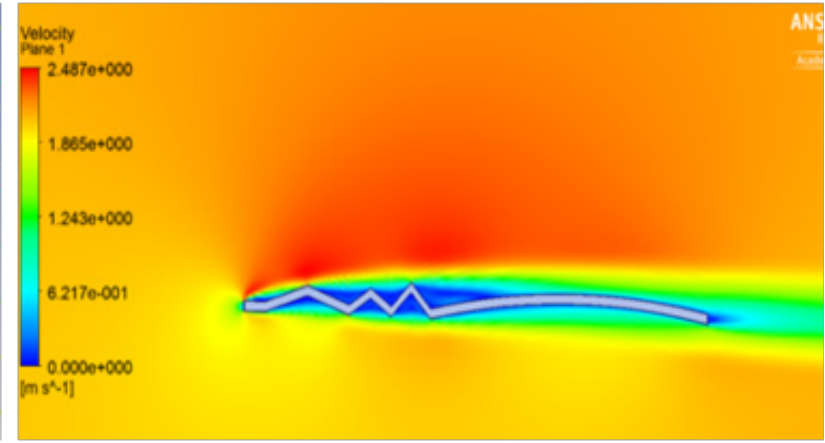

(f)

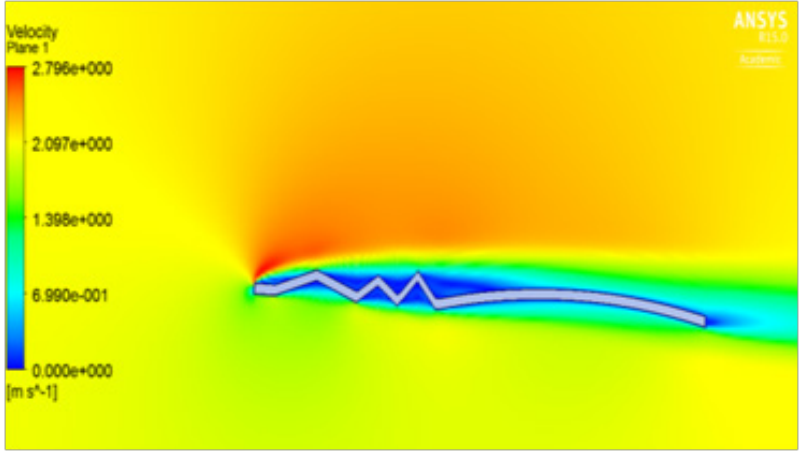

(h) 


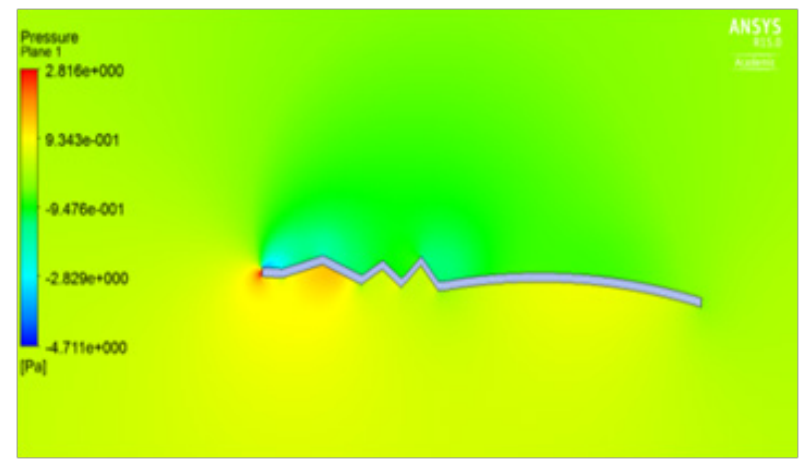

(i)

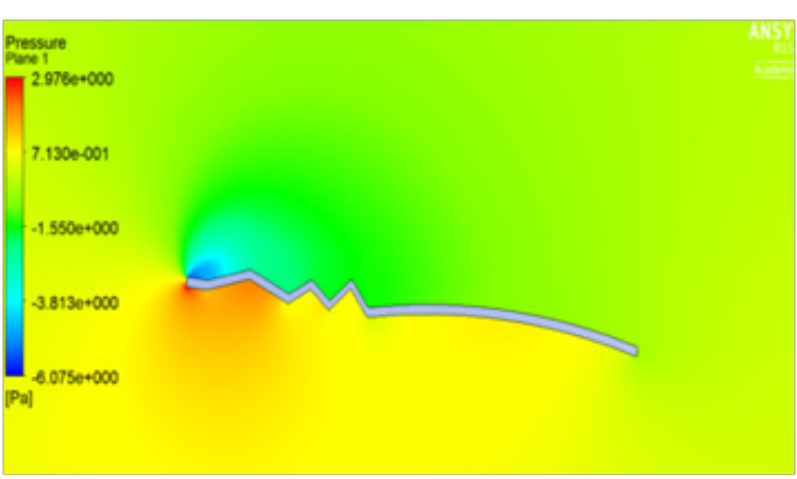

$(k)$

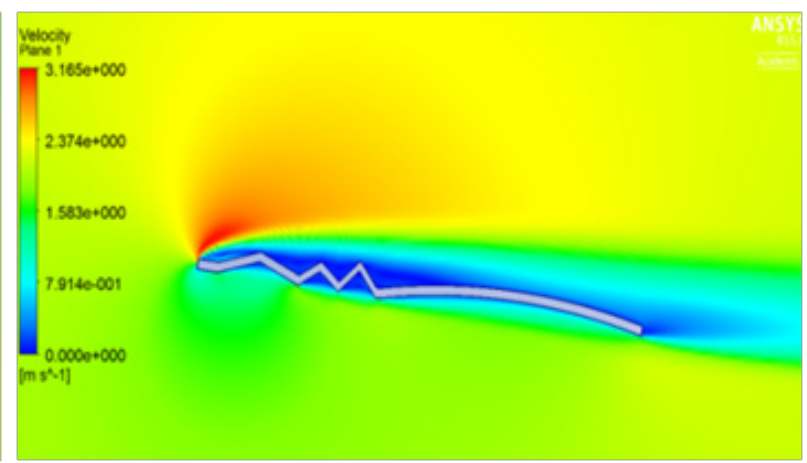

(j)

Figure 5 (a) Pressure plot at $\alpha=0^{\circ}$ (b) velocity plot at $\alpha=0^{\circ}$ (c) velocity pathline at $\alpha=0^{\circ}$ (d) Turbulence contour at $\alpha=0^{\circ}$ (e) velocity vector showing reverse flow at $\alpha=0^{\circ}$ (f) velocity plot at $\alpha=2^{\circ}$ (g) Pressure plot at $\alpha=2$ (h) velocity plot at $\alpha=4^{\circ}$ (i) Pressure plot at $\alpha=4^{\circ}$ (j) velocity plot at $\alpha=8^{\circ}$ (k) Pressure plot at $\alpha=8^{\circ}$.

\section{CFD analysis results of flat plate}

Since, the flat plate is put in a flow with zero incidences; the flow at the plate surface is slowed down because of the surface friction.

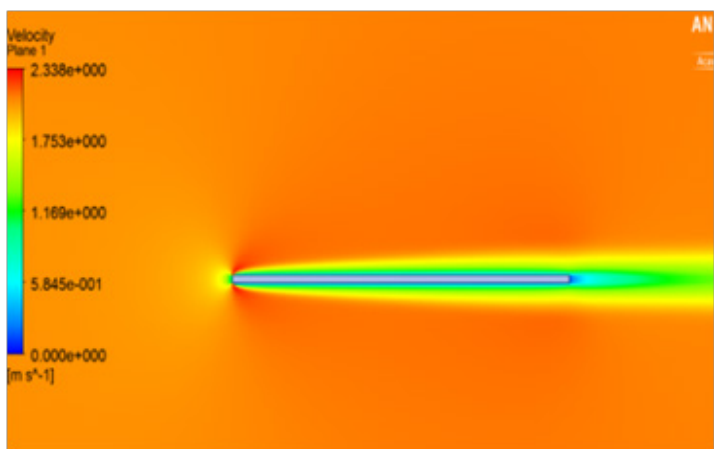

(a)

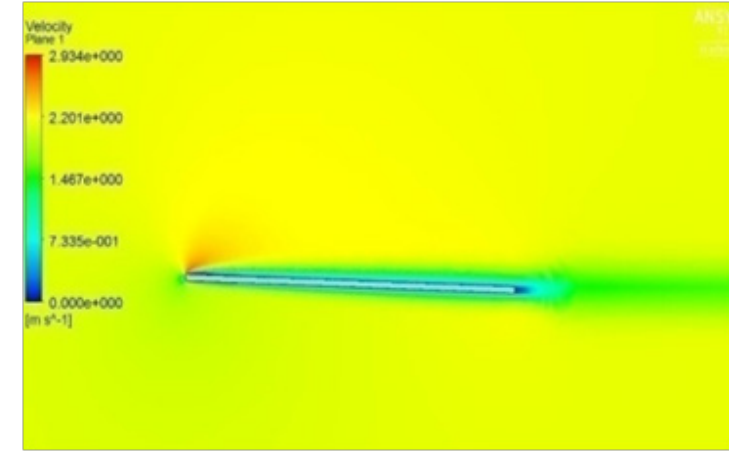

(c)
This region of slowed down flow becomes even larger and produces skin-friction drag which increases with the increase in angle of attack as shown in Figure $6 .^{6}$

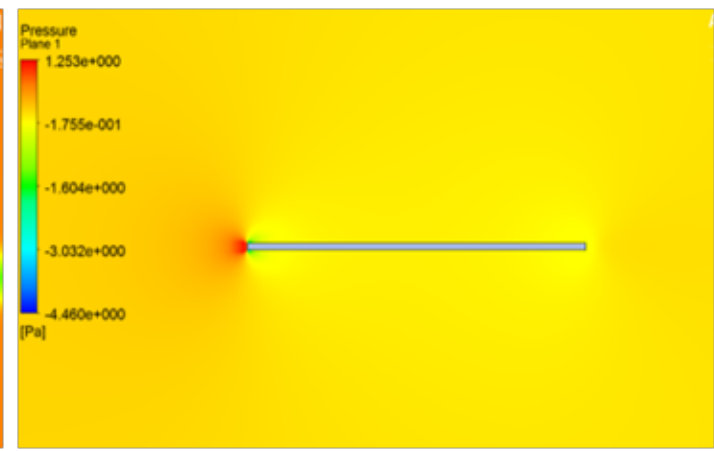

(b)

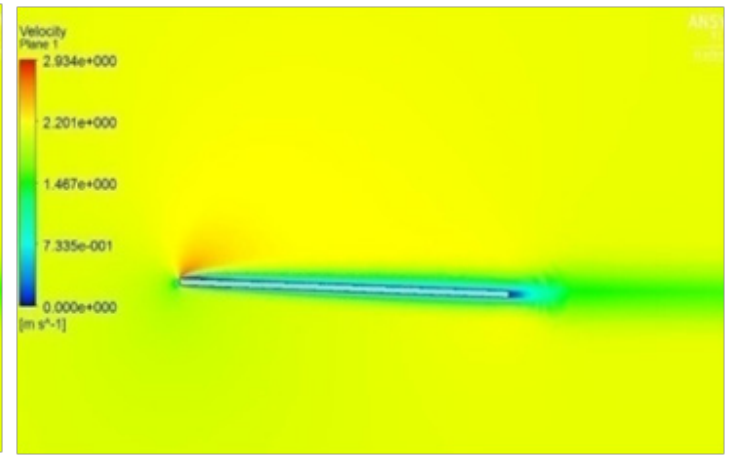

(d)

Citation: Khan A, Padhy C.Aerodynamic and structural analysis of bio-mimetic corrugated wing. Fluid Mech Res Int J. 2019;3(2):6I-67. 


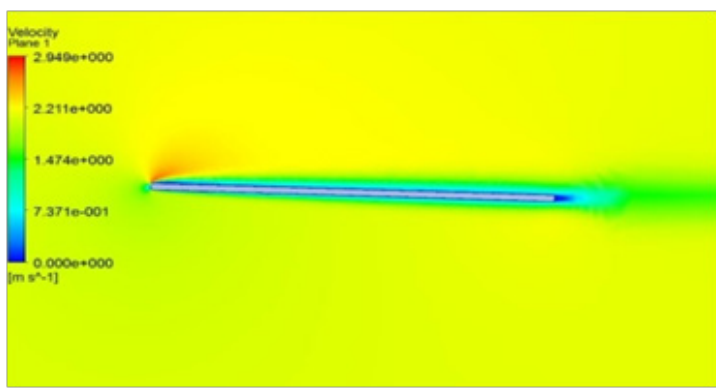

(e)

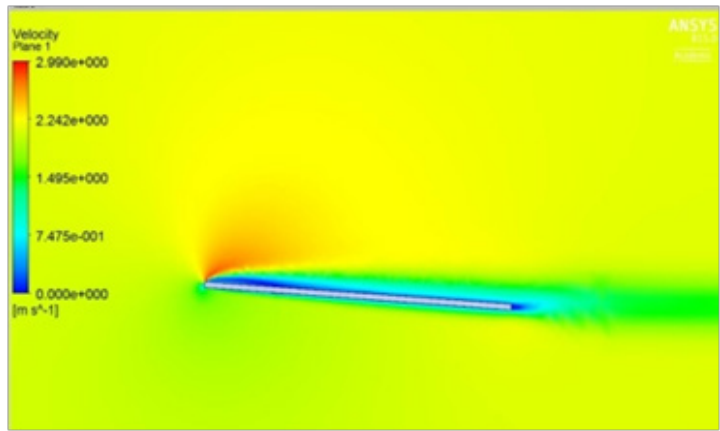

(g)

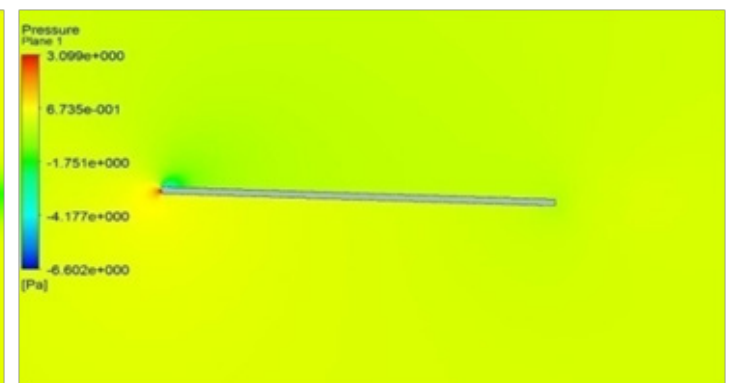

$(f)$

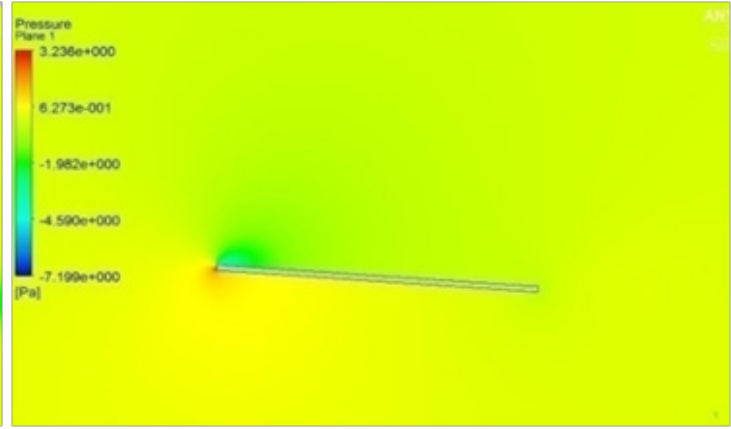

(h)

Figure 6 (a) velocity at $\alpha=0^{\circ}$ (b) pressure distribution at $\alpha=0^{\circ}$ (c) velocity distribution at $\alpha=2^{\circ}$ (d) pressure distribution at $\alpha=2^{\circ}$ (e) velocity distribution at $\alpha=4^{\circ}$ (f) pressure distribution at $\alpha=4^{\circ}$ (g) velocity distribution at $\alpha=8^{\circ}$ (h) pressure distribution at $\alpha=8^{\circ}$.

\section{Result comparison}

The computational results of the corrugated wing and flat plate are compared at different angle of attack i.e. $\left(0^{\circ}, 2^{\circ}, 4^{\circ}\right.$ and $\left.8^{\circ}\right)$. The value of $C_{L}, C_{D}$ and $L / D$ is calculated and plotted in terms of graph Table 2. ${ }^{7-9}$

Table 2 Comparison table

\begin{tabular}{llll}
\hline Wing type & $C L$ at $\left(\alpha=0^{\circ}, 2^{\circ}, 4^{\circ}, 8^{\circ}\right)$ & $C D$ at $\left(\alpha=0^{\circ}, 2^{\circ}, 4^{\circ}, 8^{\circ}\right)$ & $C_{L} / C_{D}$ at $\left(\alpha=0^{\circ}, 2^{\circ}, 4^{\circ}, 8^{\circ}\right)$ \\
\hline \multirow{3}{*}{ a Cor-wing } & $0^{\circ}-0.2177$ & $0^{\circ}-0.0670$ & $0^{\circ}-3.25$ \\
& $2^{\circ}-0.4340$ & $2^{\circ}-0.0696$ & $2^{\circ}-6.229$ \\
& $4^{\circ}-0.6327$ & $4^{\circ}-0.0797$ & $4^{\circ}-7.933$ \\
& $8^{\circ}-0.9465$ & $8^{\circ}-0.1232$ & $8^{\circ}-7.677$ \\
& $0^{\circ}-0$ & $0^{\circ}-0.0347$ & $0^{\circ}-0$ \\
b Flat Plate & $2^{\circ}-0.2209$ & $2^{\circ}-0.0522$ & $2^{\circ}-4.12$ \\
& $4^{\circ}-0.2539$ & $4^{\circ}-0.05565$ & $4^{\circ}-4.56$ \\
& $8^{\circ}-0.4412$ & $8^{\circ}-0.0683$ & $8^{\circ}-5.45$
\end{tabular}

The graph in Figure 7, which is known as the lift curve, shows that as the angle of attack increases the curve for corrugated aerofoil is linear over a considerable range giving high lift coefficient compared to flat plate. In Figure 8 it is observed that initially coefficient of drag decreases up to $6^{\circ}$ angle of attack and then it increases after reaching the stalling point at a particular speed. The graph in Figure 9 clearly shows that the performance of corrugated aerofoil is much better compared to its counterpart.

\section{Static structural analysis}

We carried out the structural analysis for the corrugated model using the ANSYS Mechanical APDL 15.0 software. The Young's modulus or modulus of elasticity is considered as, $\mathrm{E}=6.1 \mathrm{GPa}$ and Poisson's ratio, $v=0.49$ and the density, $\rho=1200 \mathrm{~kg} / \mathrm{m}^{3}$. The corrugated model section is considered as a beam and is restricted to all degrees of freedom at the leading edge. The load applied on the beam is considered as the pressure force and is distributed evenly on the entire beam surface.

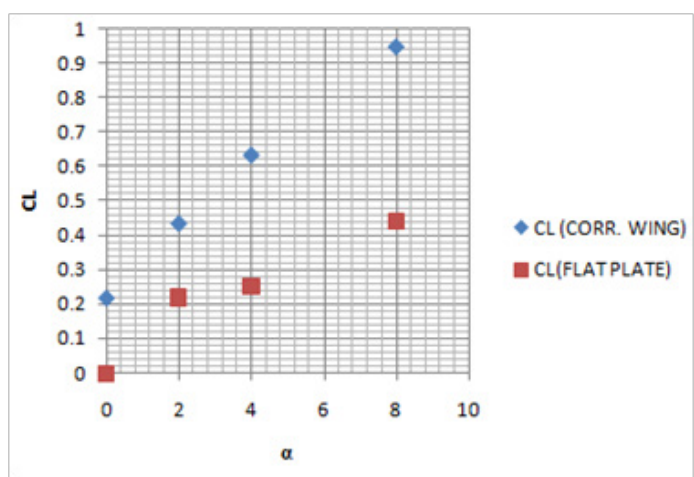

Figure $7 C_{L}$ vs $\alpha$. 

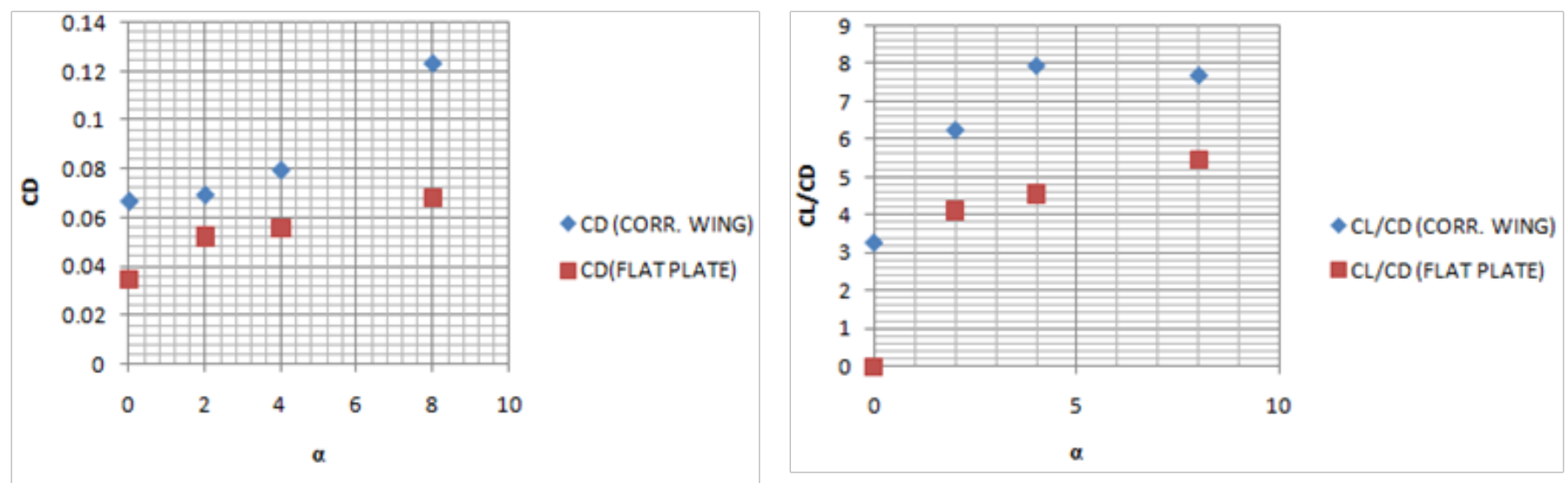

Figure $8 C_{D} v s \alpha$

Figure $9 C_{L} / C_{D} V S \alpha$
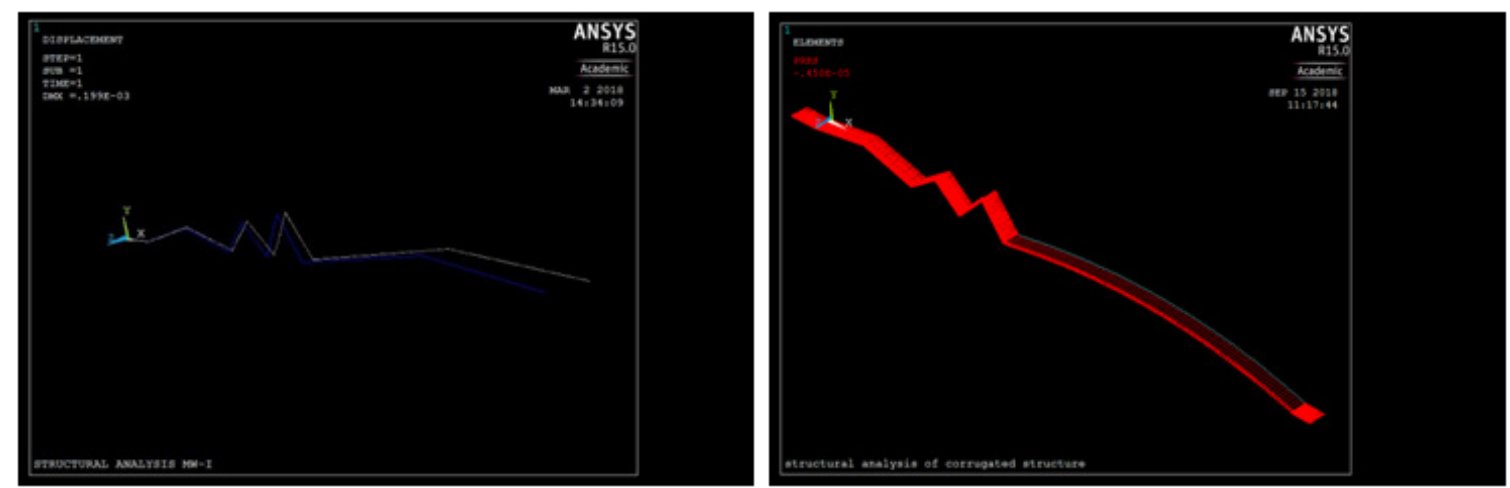

(a)

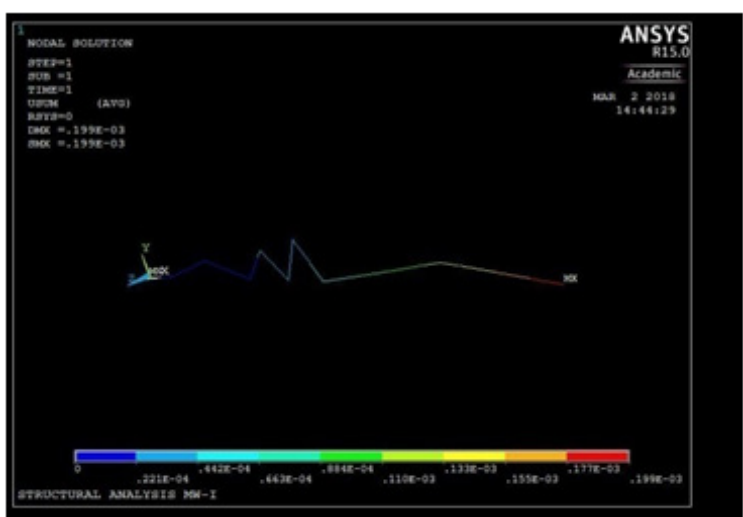

(b)

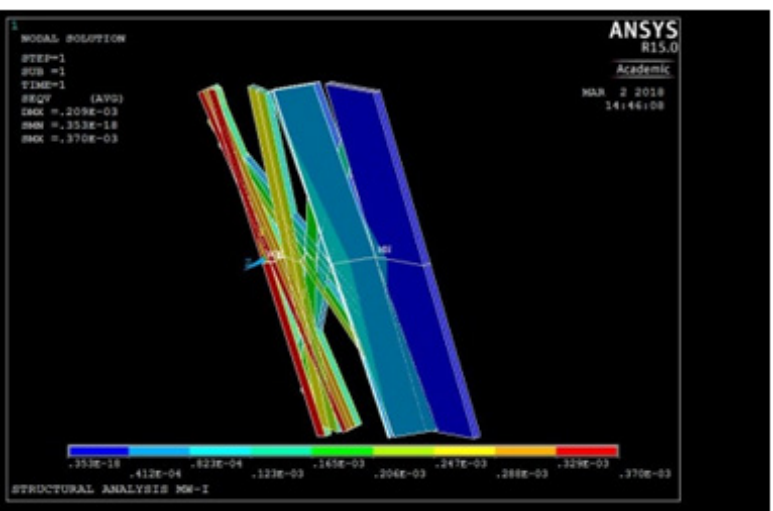

(c)

(d)

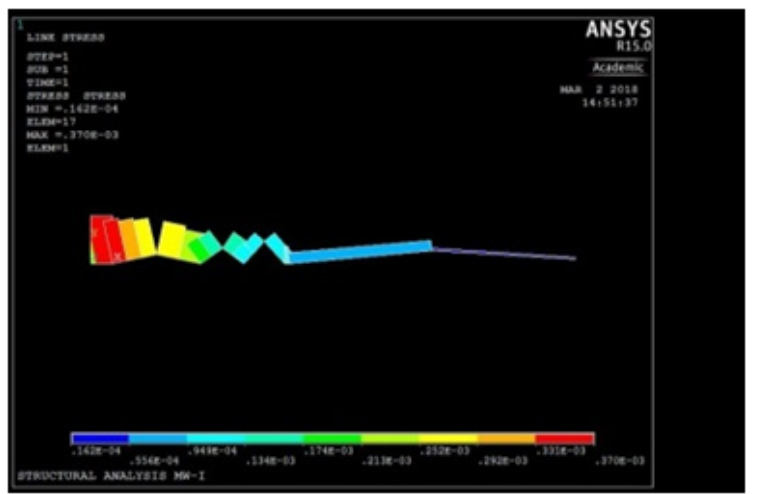

(e)

Figure 10 (a) Deformed+Un-deformed shape of corrugated wing (b) Pressure distribution (c) Displacement in z-direction (d)Von-misses stress (e)strain in z-direction.

Citation: Khan A, Padhy C.Aerodynamic and structural analysis of bio-mimetic corrugated wing. Fluid Mech Res Int J. 2019;3(2):6I-67. DOI: 10.15406/fmrii.2019.03.00053 
From the Figure 10(b), we can see that the maximum displacement in $\mathrm{z}$ direction at the trailing edge is $0.199 \times 10^{-4} \mathrm{~mm}$ and the minimum deflection is at the leading edge. The maximum Von-misses stress obtained is $0.370 \times 10^{-3} \mathrm{~N} / \mathrm{mm}^{2}$ at the leading edge or at the root of the corrugated aerofoil.

\section{Conclusion}

It is found that the design criteria being used and simulation carried out on the corrugated wing at low Reynolds number is giving much better results. The flow separates from the corrugation instantly reattaches to the upper surface and get trapped in the valleys which delays flow separation. The aerodynamic performance i.e. L/D ratio of the corrugated wing increases which improves the gliding of the dragonfly. ${ }^{10-12}$ The structural analysis shows that the corrugated structure can withstand inertial loads and shearing. In corrugated wing structure if one member fails the other member can withstand that load which prevents the dragonfly wing fracture during flight condition. The wing deformation during down stroke due to camber causes the wing being more rigid to forces applied from the concave side than from the convex side resulting in high stiffer wings and more precised for flapping MAVs. Hence, it is concluded that the corrugated wing can withstand both aerodynamic and structural load which is most suitable for future MAVs and UAVs.

\section{Acknowledgments}

None.

\section{Conflicts of interest}

The authors declares there is no conflicts of interest.

\section{References}

1. D Alexander. Unusual phase relationships between the forewing and hind wing in flying dragonflies. Journal of Experimental Biology. vol.1984;109:379-383.
2. Kesel AB. Aerodynamic characteristics of dragonfly wing sections compared with technical aerofoil. J Exp Biol. 2000;203(20):3125-3135.

3. Okamoto M, Yasuda K, Azuma A. Aerodynamic Characteristics of the wings and the Body of a dragonfly. Journal of experimental biology. 1996;199:281-294.

4. Rees CJC. Aerodynamic properties of an insect wing section and a smooth aerofoil compared. Nature. 1975;258(5531):141-142.

5. Liu H, Ellington $\mathrm{C}$, Kawachi K, et al. A computational fluid dynamic study of hawk moth hovering. J Exp Biol. 1998;201(4):461-477.

6. Wu JH, Sun M. Unsteady aerodynamic forces of a flapping wing. $J$ Exp Biol. 2004;207(7):1137-1150.

7. Chen YH, Skote M. Study of lift enhancing mechanisms via comparison of two distinct flapping patterns in the dragonfly sympetrum flaveolum. Physics of Fluids. 2015;27(3):033604.

8. David Elie Levy, Avraham Seifert. Simplified dragonfly airfoil aerodynamics at Reynolds numbers below 8000. Physics of fluids. 2009;21(7):071901.

9. Combes SA, Daniel TL. Flexural stiffness in insect wings II Spatial distribution and dynamic wing bending. J Exp Biol. 2003;206(17):29892997.

10. Wootton RJ, Herbert RC, Young PG, et al. Approaches to the structural modelling of insect wings. Phil Trans $R$ Soc Biol Sci. 2003;358(1437):1577-1587.

11. Lazos BS. Biologically Inspired Fixed-Wing Configuration Studies. Journal of Aircraft. 2005;42(5).

12. Wakeling JM, Ellington CP. Dragonfly Flight I. Gliding Flight and Steady-state Aerodynamic Forces. Journal of Experimental Biology. 1997;200(3):543-556. 